\title{
Remote Synchronization Experiments for Quasi-Senith Satellite System Using Current Geostationary Satellites
}

\author{
Toshiaki Iwata, ${ }^{1}$ Tomonari Suzuyama, ${ }^{2}$ Michito Imae, ${ }^{2}$ and Yuji Hashibe ${ }^{3}$ \\ ${ }^{1}$ Collaborative Research Team for Verification, Kansai Collaborative Center, National Institute of Advanced Industrial Science and \\ Technology (AIST), 1-8-31 Midorigaoka, Ikeda, Osaka 563-8577, Japan \\ ${ }^{2}$ Laboratory of Frequency Measurement Systems, National Metrology Institute of Japan, National Institute of Advanced Industrial \\ Science and Technology (AIST), 1-1-1 Umezono, Central 3, Tsukuba, Ibaraki 305-8563, Japan \\ ${ }^{3}$ Space Engineering Development Co. Ltd., 5-62-1 Nakano, eDC-building, Nakano-ku, Tokyo 164-0001, Japan
}

Correspondence should be addressed to Toshiaki Iwata, totty.iwata@aist.go.jp

Received 3 February 2010; Accepted 30 April 2010

Academic Editor: Patrizia Tavella

Copyright (c) 2010 Toshiaki Iwata et al. This is an open access article distributed under the Creative Commons Attribution License, which permits unrestricted use, distribution, and reproduction in any medium, provided the original work is properly cited.

\begin{abstract}
The remote synchronization system for the onboard crystal oscillator (RESSOX) realizes accurate synchronization between an atomic clock at a ground station and the QZSS onboard crystal oscillator, reduces overall cost and satellite power consumption, as well as onboard weight and volume, and is expected to have a longer lifetime than a system with onboard atomic clocks. Since a QZSS does not yet exist, we have been conducting synchronization experiments using geostationary earth orbit satellites (JCSAT$1 \mathrm{~B}$ or Intelsat-4) to confirm that RESSOX is an excellent system for timing synchronization. JCSAT-1B, the elevation angle of which is 46.5 degrees at our institute, is little affected by tropospheric delay, whereas Intelsat- 4 , the elevation angle of which is 7.9 degrees, is significantly affected. The experimental setup and the results of uplink experiments and feedback experiments using mainly Intelsat- 4 are presented. The results show that synchronization within $10 \mathrm{~ns}$ is realized.
\end{abstract}

\section{Introduction}

The Quasi-Senith Satellite System (QZSS) has been under development as a Japanese space project since 2003, and its mission is navigation and positioning [1]. Its constellation consists of three satellites orbiting on inclined orbital planes with a geosynchronous period. The QZSS utilizes a highly inclined orbit because of the high visibility over high-latitude regions. In the case of the QZSS, at least one satellite is highly visible near the zenith at any time from Japan. Therefore, users can always receive navigation signals from at least one of the QZSs near the zenith. The first satellite of the QZSS will be launched in the summer of 2010 .

In general, global navigation satellite systems (GNSSs), such as the GPS of the USA, GLONASS of Russia, GALILEO of Europe and COMPASS of China, are equipped with onboard atomic frequency standards that are used as the time reference. This is because (1) atomic frequency standards have good midterm and long-term stability, (2) the orbit of satellites makes monitoring from only one ground station impossible, (3) these satellite systems are used for military missions and are therefore expected to operate even if ground stations are destroyed, and (4) these systems consist of many satellites, making the continuous control of each satellite with many antennae difficult. However, onboard atomic clocks have the following disadvantages: they are bulky, expensive to manufacture and launch, and powerdemanding. Moreover, they are one of the main factors contributing to the reduction of satellite lifetime.

The following has been taken into consideration in the design of the QZSS as a civilian navigation system: (1) some crystal oscillators have better short-term stability than atomic clocks [2], (2) 24-hour control from one station is possible if the location of the control station is appropriate, for example, Okinawa, Japan, and (3) the number of satellites is assumed to be only three. Given these considerations, we have developed a remote synchronization system for the onboard crystal oscillator (RESSOX), which does not require onboard atomic clocks. In the case of RESSOX, the modification of the control algorithm after 
launch is easy because it is basically a ground technology. The target synchronization accuracy of RESSOX is set at $10 \mathrm{~ns}$ (peak-to-peak) and the target stability is $1 \times 10^{-13}$ at $100,000 \mathrm{~s}$. These targets were determined on the basis of the synchronization performance between GPS time and UTC (USNO) [3] and the long-term stability performance of onboard cesium atomic clocks [4]. Similar research has been conducted by Giunta et al. [5], however, their goal was a time and frequency dissemination system using GEO/MEO/LEO satellites and TDMA.

RESSOX ground experiments and computer simulations have been conducted since 2003. Primary experimental results obtained using navigation signals are detailed in our previous papers [6-9]. We have developed a feedback method that uses multiple navigation signals of the QZSS, and found that we do not need precise orbit information or any estimation of delays, such as those caused by the ionosphere and troposphere, to realize RESSOX technology.

In a practical sense, the QZSS will be equipped with two rubidium atomic standards. RESSOX research is cutting a path to future technology.

Since no QZSS exists at the moment, we validate RESSOX techniques using geostationary earth orbit (GEO) satellites as an actual space system. Two GEO satellites, JCSAT-1B (elevation angle of 46.5 degrees as viewed from our institute) and Intelsat-4 (formerly PAS-4; elevation angle of 7.9 degrees as viewed from our institute), have been chosen as relay satellites because the elevation angle of the QZSS at Okinawa is expected to be between 10 and 90 degrees.

\section{RESSOX Overview}

Figure 1 shows the schematic of RESSOX. In order to realize RESSOX, it is essential to identify the error factors and the feedback mechanism by measuring the delay at the ground station. The former is related to the estimation of error and delay using models, and is considered to be a feedforward loop. The RESSOX control signal includes the time information of the ground atomic clock, and is advanced to compensate the transmission delay. Thus, the RESSOX control signal is synchronized with the ground atomic clock when it arrives at the QZSS. The onboard crystal oscillator is controlled to follow the RESSOX control signal.

Navigation signals are broadcast from the QZSS on the basis of the crystal oscillator time. On the ground, the QZSS/GPS receiver measures the pseudoranges of the navigation signals. The difference between measured and estimated pseudoranges is used to adjust the RESSOX control signal. This is considered as feedback control.

The error and delay models in the feed-forward loop are for delays in the ground station and in the satellite, tropospheric delay, ionospheric delay, delay due to distance (orbit estimation), delay due to relativity effects, and errors caused by Earth's motion, such as daily rotation, nutation, and precession. These problems were discussed in our previous paper [6]. However, if multiple navigation signals are used for feedback, the use of the delay models of the troposphere and ionosphere becomes unnecessary $[8,9]$.

\section{Remote Synchronization Experiments Using GEO Satellites}

In this section, we will introduce the equipment used in the experiments. Then, we will discuss briefly the experimental results of JCSAT-1B and explain in detail the results of preliminary experiments and experiments using Intelsat- 4 .

3.1. Experimental Setup. The experimental setup on the ground is common to both cases of JCSAT-1B and Intelsat4 . The only differences are the satellites, the antennae, and the up/down-converters used to communicate with them. The concept of the GEO satellite experiments is shown in Figure 2. Two ground sites were provided. One is the GROUND ground site with the hydrogen maser and the other is the GROUND QZSS site with the crystal oscillator. The GEO satellite reflects signals from each site like a mirror; one round trip to and from the GEO satellite corresponds to one way to the QZSS. In this figure, the two sites appear to be at different places, but in the actual experiment, both sites were at the same location.

The block diagram of the experiments is shown in Figure 3. The following are the apparatuses used at the GROUND ground site.

Hydrogen Maser (H-Maser). The $\mathrm{H}$-maser used in the experiments is an active hydrogen maser (RH-401A) manufactured by Anritsu and is one of the atomic clocks used to generate national time and frequency standards in Japan (UTC (NMIJ)). It was used as the reference atomic clock for the apparatuses at the GROUND ground site in the experiments.

Transmitting Time Adjuster (TTA). TTA generates the advanced time of the $\mathrm{H}$-maser and provides inverse Doppler effects to change the frequency of the two-way satellite time and frequency transfer (TWSTFT) modem. It is based on the wavefront clock principle [10]. In the experiments, TTA was used to compensate the propagation time of the RESSOX control signal by means of the estimated propagation time that was computed with our simulators beforehand. This corresponds to the feed-forward control of RESSOX. The feedback commands generated by the timing controller are also received.

Modem (T). Modem (T) is one of the three TWSTFT modems used in the experiments ("T" stands for "transmitting"). This TWSTFT modem has one transmitting channel that transmits a modulation wave of its own reference with the pseudonoise (PN) code. It also has two receiving channels for comparing the time difference between the time of the input clock signal (1 pps and $10 \mathrm{MHz}$ ) and that of the received PN signal. In the experiments, modem (T) was referenced to the time signal of TTA and the RESSOX control signal was transmitted to modem (TR) of the QZSS site via the GEO satellite.

Modem (R). Modem (R) is also one of the three TWSTFT modems used in the experiments, has the $\mathrm{H}$-maser as the 


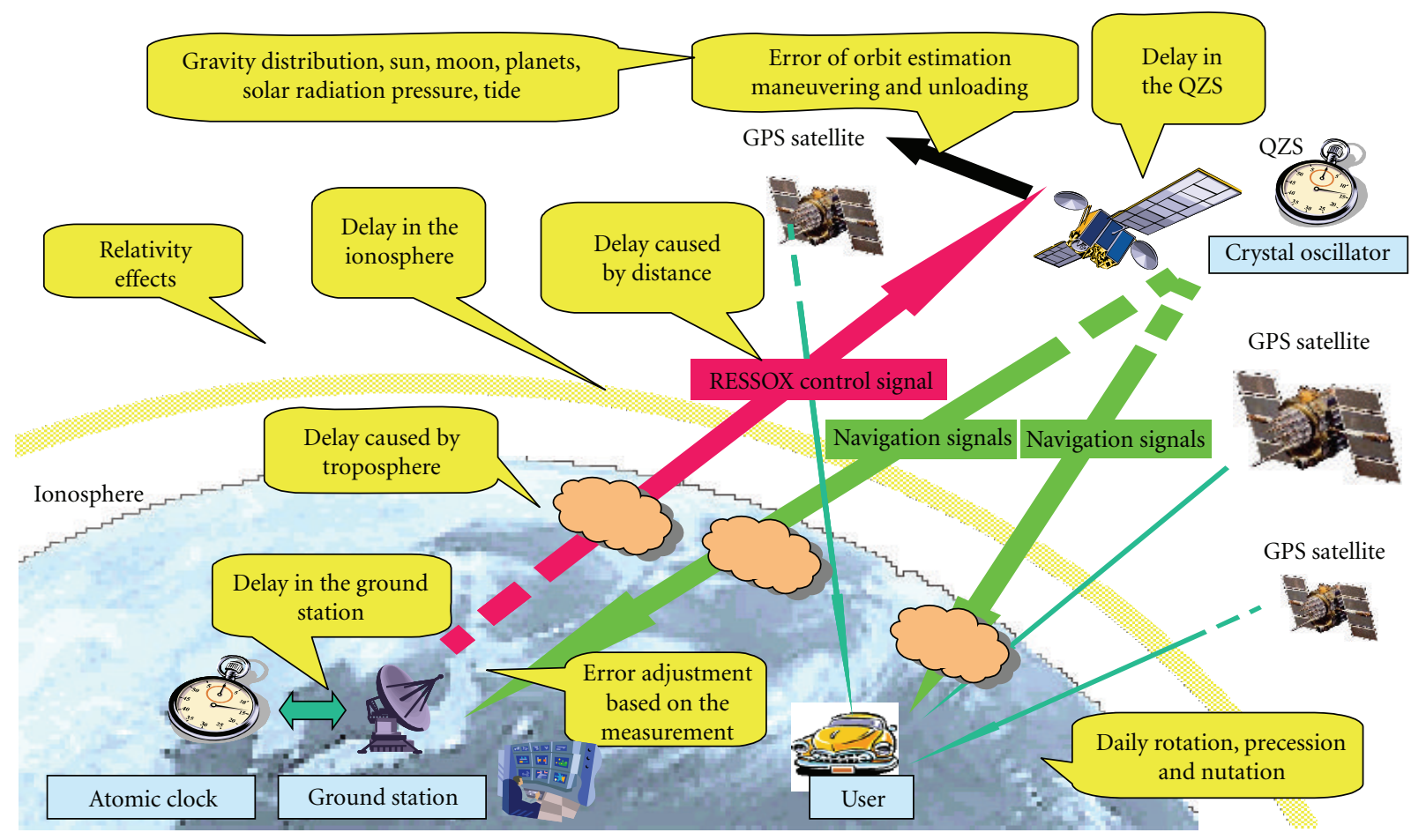

FIGURE 1: RESSOX schematic.

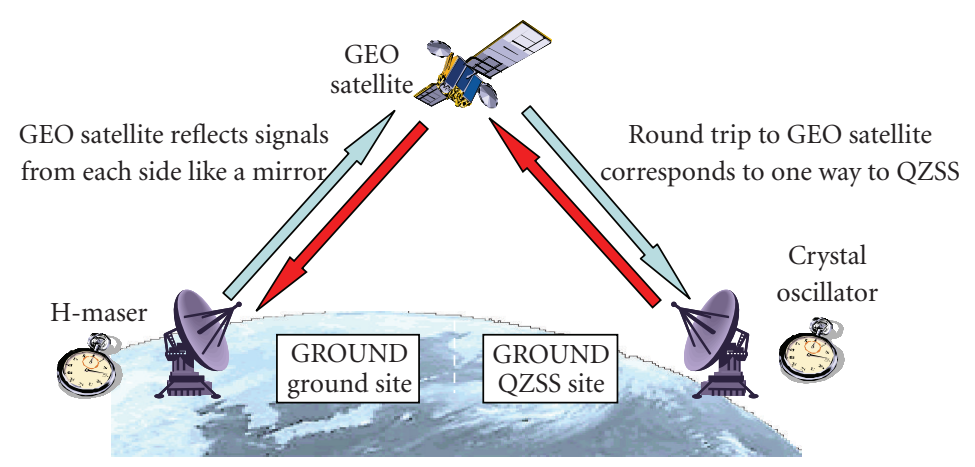

FIgURE 2: Concept of GEO satellite experiments.

reference time, and corresponds to the QZSS/GPS receiver that receives navigation signals, such as L1-, L2- and L5bands, in an actual RESSOX ("R" stands for "receiving"). From the QZSS site, it receives the PN-modulated time signal of a crystal oscillator sent from modem (TR) via the GEO satellite. This signal corresponds to the navigation signals of the QZSS in an actual RESSOX. The time difference was compared, as explained earlier, and the propagation time that corresponds to a pseudorange of a navigation signal in the actual RESSOX was observed.

Timing Controller. The timing controller is a Windows XP PC. The timing controller generates an estimation of the uplink propagation time and feedback commands in the form of estimation errors of downlink propagation time, that is, the difference between the propagation time observed with modem $(\mathrm{R})$ and the propagation time estimated with our simulators.

Apparatuses for the GROUND QZSS site are listed as follows.

Crystal Oscillator. A very stable BVA-type oven-controlled crystal oscillator (OCXO) manufactured by Oscilloquartz S.A. (OCXO 8607) is used for synchronization with the Hmaser. The Allan deviation of the crystal oscillator used in the experiments is shown in Figure 4. Red and black lines show the results obtained using the $\mathrm{H}$-maser and another voltage-controlled crystal oscillator (VCXO) as reference, respectively. As VCXO has better short-term stability, the latter results are better than the former when the averaging time is less than $10 \mathrm{~s}$. The H-maser's stability was $5.0 \times 10^{-13}$ at $1 \mathrm{~s}$, and in our experiments, the signal was distributed 


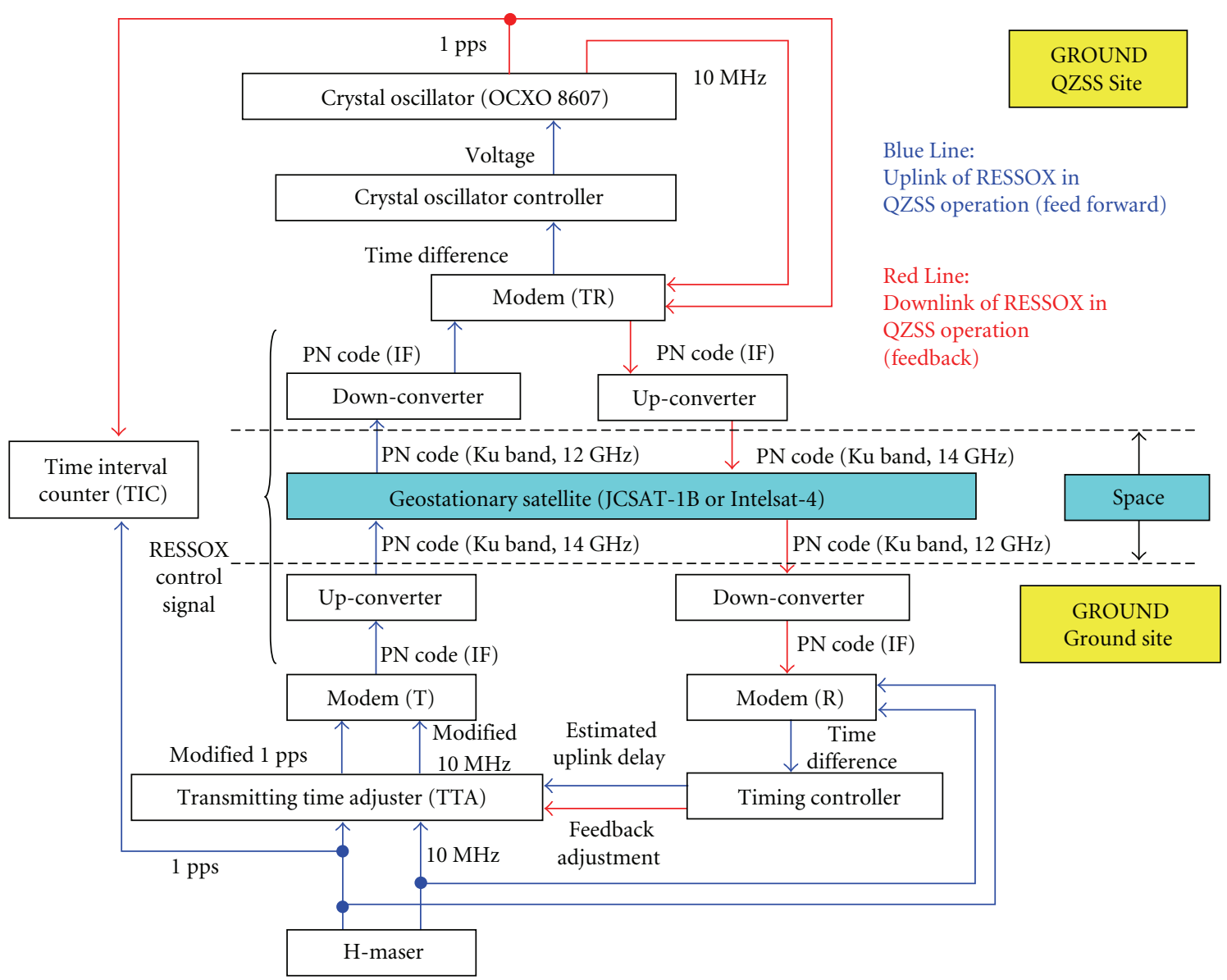

FIGURE 3: Schematic for RESSOX experiments using geostationary satellites.

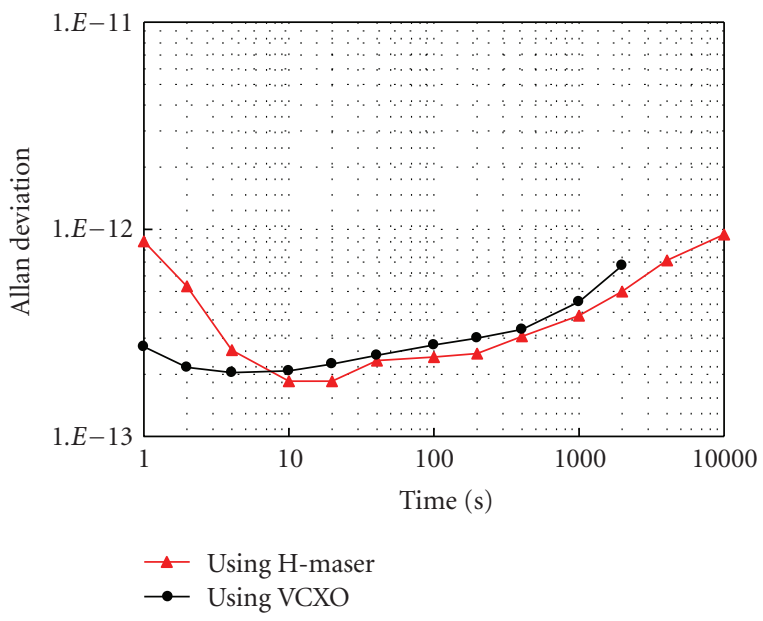

FIgURE 4: Allan deviation of OCXO 8607.

by a distribution amplifier and transmitted about $100 \mathrm{~m}$. Therefore, we consider that the stability of $8.5 \times 10^{-13}$ at $1 \mathrm{~s}$ is satisfactory. In the experiments, OCXO 8607 was controlled to a certain offset that was intentionally given, because the time interval counter could not display negative values. Every apparatus at the GROUND QZSS site is referenced to OCXO 8607 time.
Modem (TR). Modem (TR) is one of the three TWSTFT modems used in the experiments and was referenced to OCXO 8607 ("TR" stands for "transmitting and receiving"). It receives RESSOX control signals from modem $(\mathrm{T})$ via the GEO satellite, and the time difference between OCXO 8607 time and the RESSOX control signal, which includes the clock error of OCXO 8607 and the estimation error of uplink propagation time. Concurrently, it also transmits a $\mathrm{PN}$-modulated signal, which is a substitute of the navigation signals of the QZSS, to modem (R) via the GEO satellite.

Crystal Oscillator Controller. The crystal oscillator controller, which consists of a Macintosh PC and a voltage generator, generates input voltage of the crystal oscillator to reduce the observed time difference in modem (TR). In the experiments, a kind of PI control, as described by the following equation, was used to control the crystal oscillator.

$$
\begin{aligned}
v_{k}= & \text { offset }-\frac{k_{1}}{l+1} \sum_{i=k-l}^{k}\left(t_{\text {OCXO }}-t_{\text {RESSOX }}\right)_{i} \\
& -k_{2} \sum_{i=0}^{k-1}\left(\int_{i}^{i+p}\left(t_{\text {OCXO }}-t_{\text {RESSOX }}\right)\right) d t .
\end{aligned}
$$




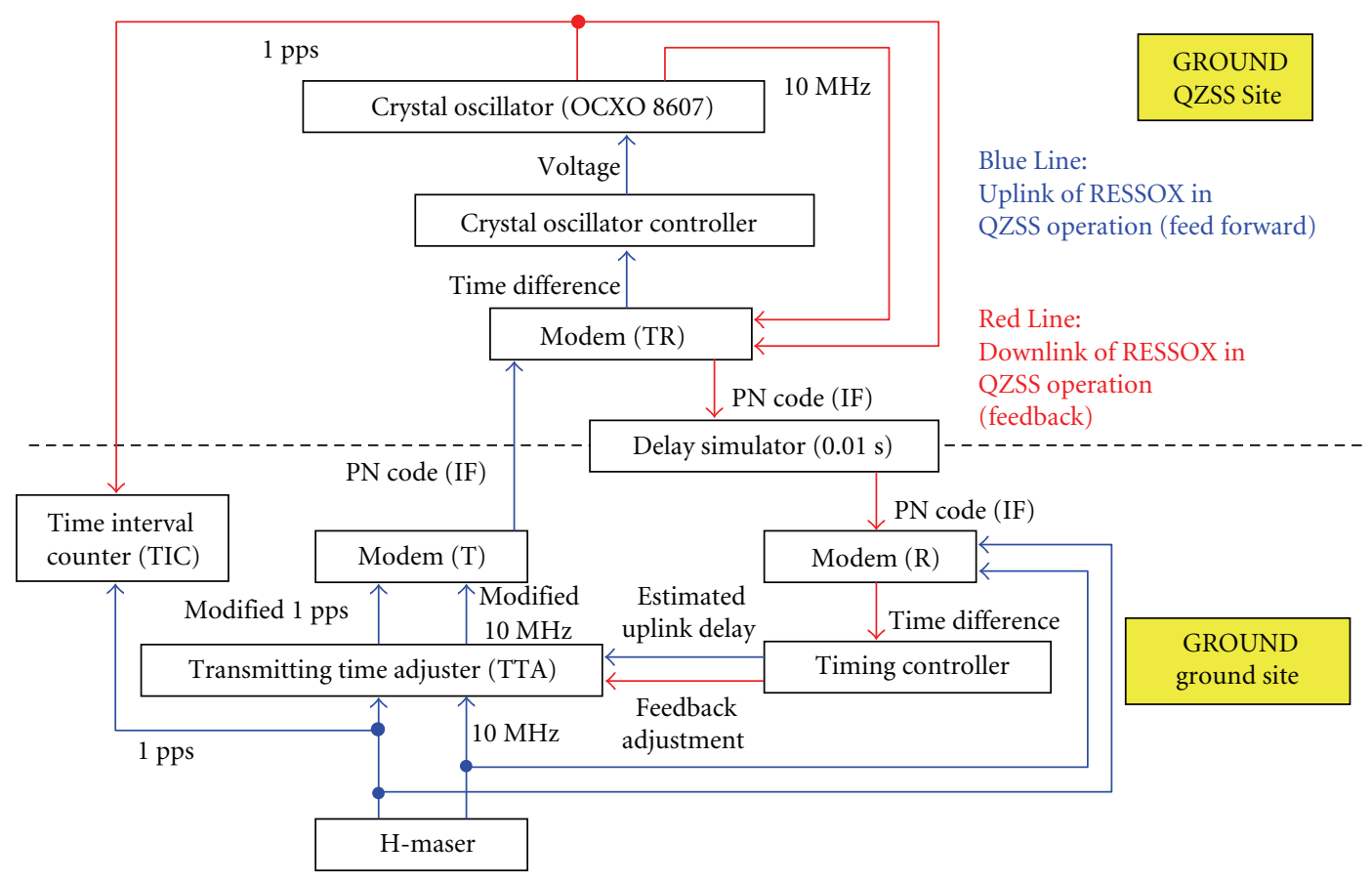

FIGURE 5: Preliminary ground experiment setup.

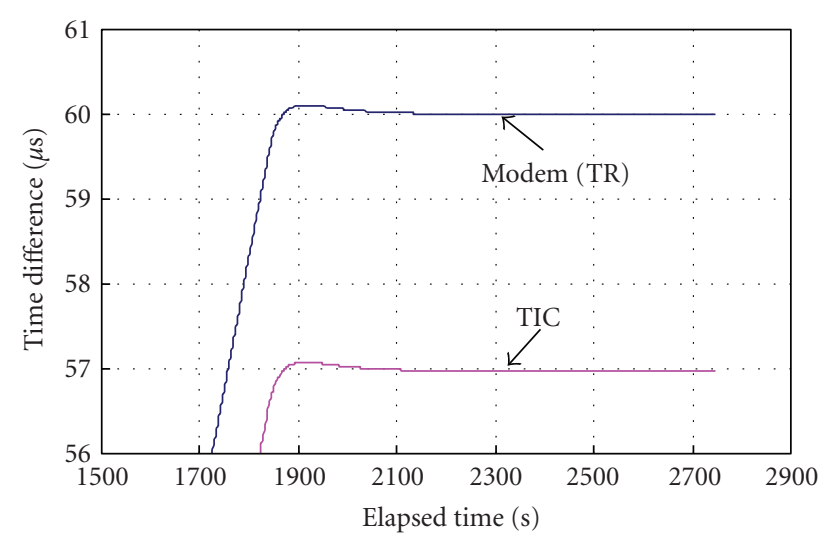

FIGURE 6: Result of feed-forward control without TTA internal delay consideration.

Here, $v_{k}$ is the $k$ th output voltage, offset $=4.945(\mathrm{~V}), k_{1}$ is a proportional gain set at $7.6 \times 10^{6}, k_{2}$ is an integral gain set at $3.0 \times 10^{4}, l$ is the number of past data values used for proportional control set at $1, k$ is the data number from the beginning, $p$ is the integral interval, which means an overlapping integral number, set at $2, t_{\mathrm{RESSOX}}$ is the time information of the received RESSOX control signal, and $t_{\text {OCXO }}$ is the time of OCXO 8607. These factors were determined by trial and error. In actual use, the integration part was effective only when the error $\left(t_{\text {OCXO }}-t_{\text {RESSOX }}\right)$ was less than $1 \mu \mathrm{s}$.

Finally, apparatuses that were not categorized at either site are listed as follows.

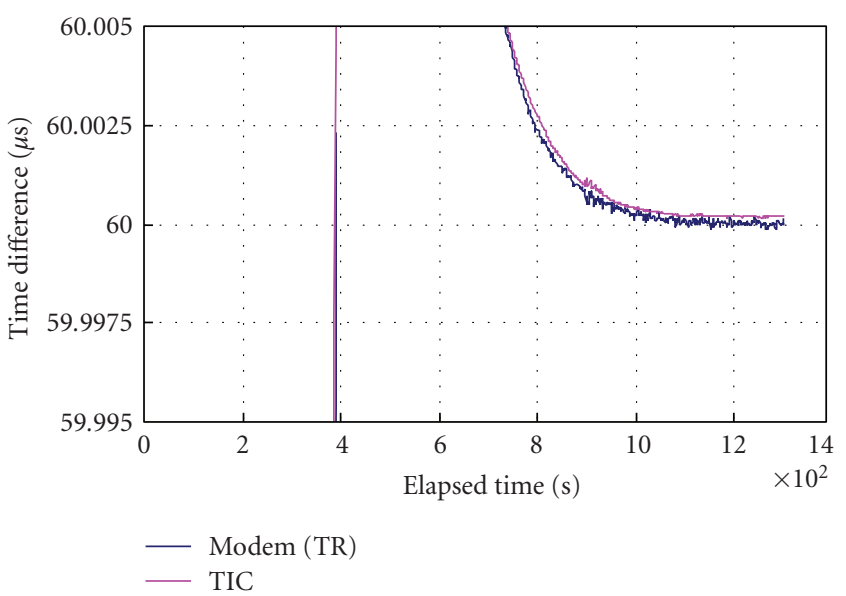

FIGURE 7: Result of feed-forward control with TTA internal delay consideration.

Time Interval Counter (TIC). TIC displays the time difference of the two clocks. It displays only positive values. In the experiments, TIC displays the time difference between the $\mathrm{H}$ maser and OCXO 8607, including the offset given in OCXO 8607. Since OCXO 8607 was implemented on the ground test bed in the experiments, TIC was used in the experiments but not in the actual QZSS operation.

GEO Satellites. GEO satellites used for the experiments are JCSAT-1B operated by JSAT Corporation and Intelsat-4 operated by Intelsat Corporation. JCSAT-1B drifts in the vicinity of the longitude of 150 degrees east, and Intelsat4 , the longitude of 72 degrees east. Frequency bands of 


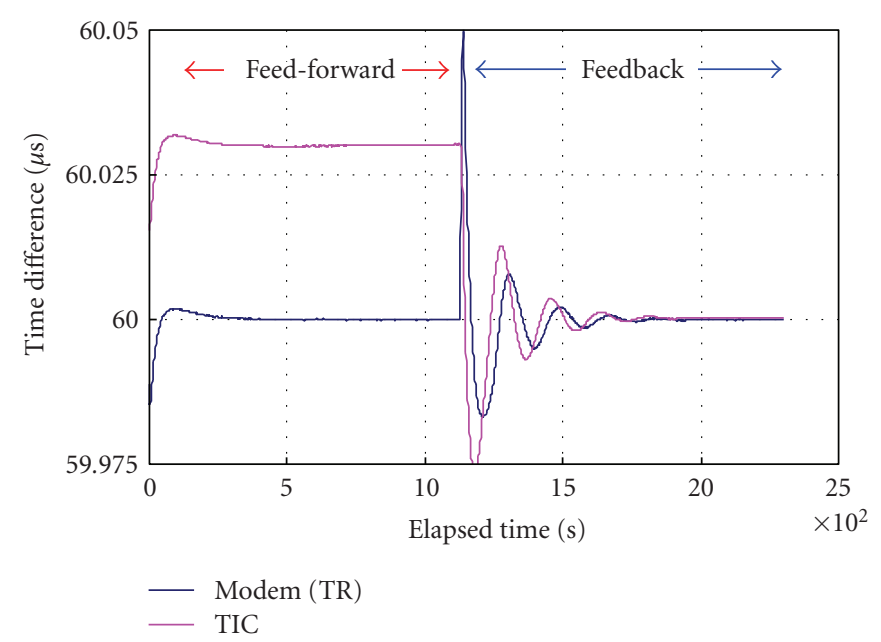

(a)

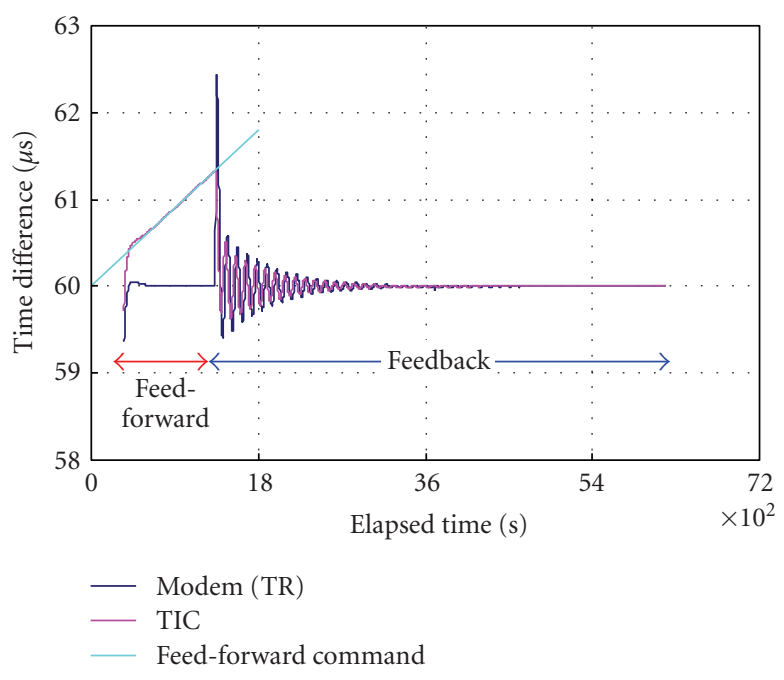

(b)

FIGURE 8: Results of feedback control in preliminary ground experiments.

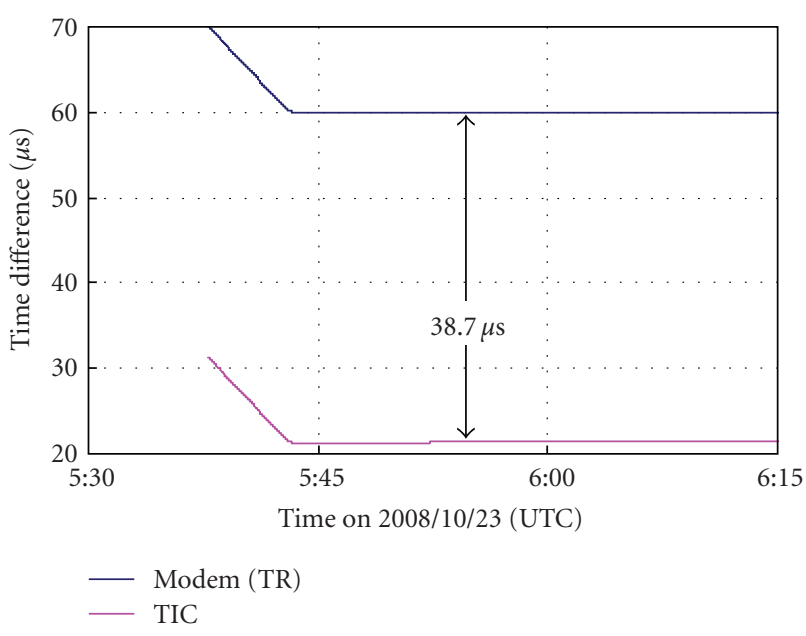

FIGURE 9: Result of feed-forward control using Intelsat-4.

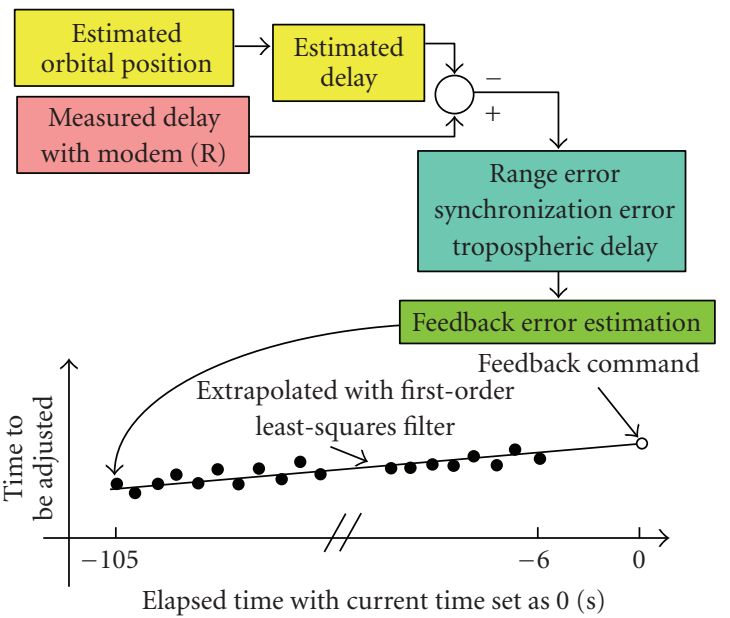

Figure 10: Feedback mechanism.

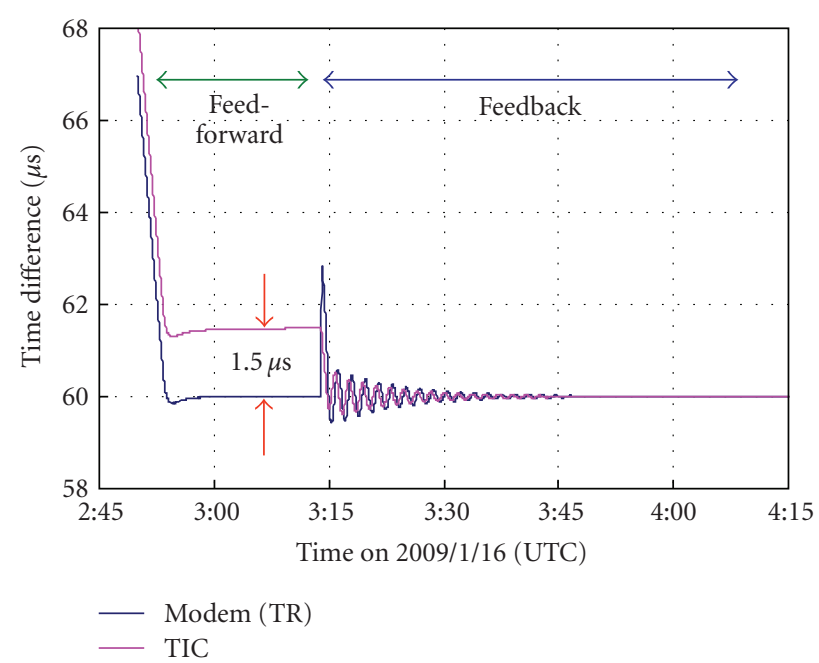

FIGURE 11: Results of feedback experiments.

14.051 GHz ( $\mathrm{Ku}$ band) and $12.303 \mathrm{GHz}$ ( $\mathrm{Ku}$ band) were used for the uplink and downlink signals of JCSAT-1B, and 14.610375 GHz (Ku band) and 12.661500 GHz (Ku band) for those of Intelsat-4, respectively. The satellites are used as a mirror to reflect the signals from the GROUND ground site to the GROUND QZSS site and vice versa.

3.2. Difference between Using QZSS and GEO Satellites. When the GEO satellite is used for RESSOX experiments, the following differences should be noted.

(1) The satellite direction is not changed. This means that dynamic changes in tropospheric and ionospheric delays are negligible when RESSOX is operated. Moreover, the multipath condition does not change during the experiments. 


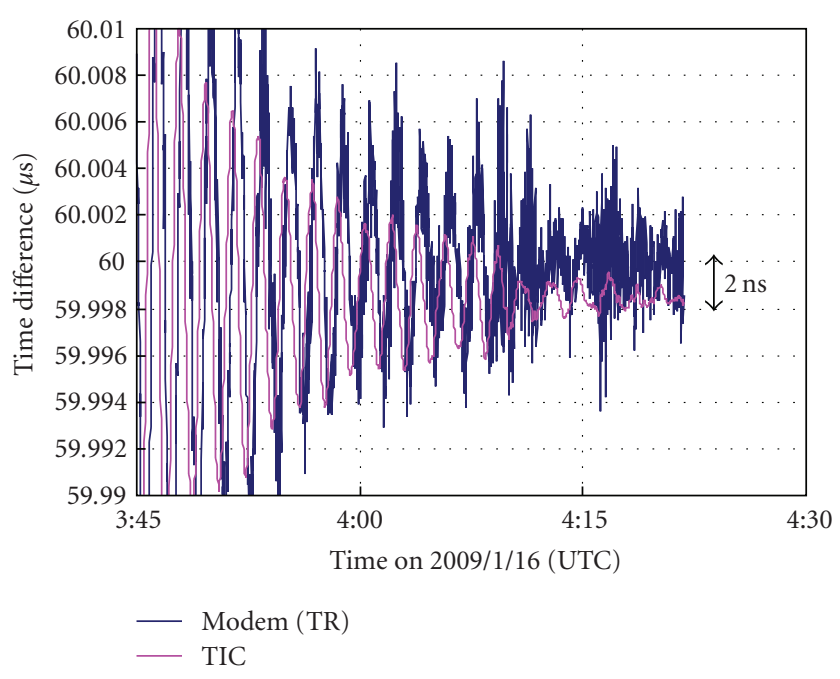

FIGURE 12: Results of feedback experiments (magnified).

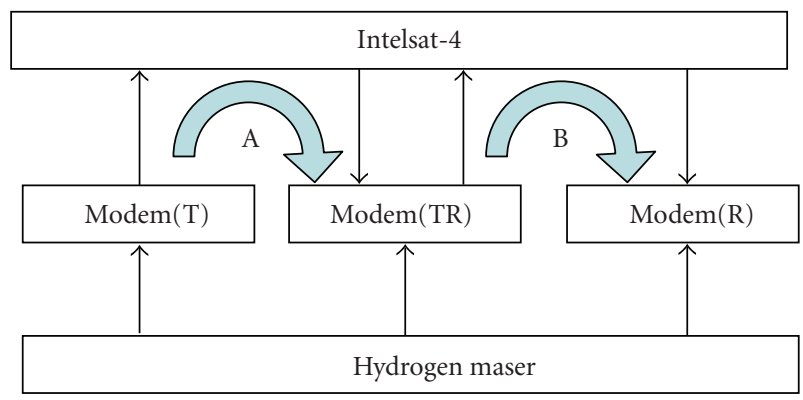

FIGURE 13: Block diagram of loop delay measurements.

(2) The downlinked signal is transmitted via the $\mathrm{Ku}$ band. This means that ionospheric delay cannot be estimated by using multiple frequencies; however, $\mathrm{Ku}$-band signals are not sensitive to ionospheric delay because of their short wavelength.

3.3. Overview of JCSAT-1B Experiments. JCSAT-1B was launched on December 3, 1997, from Guiana on the Ariane 4 rocket, and is located at 150 degrees east longitude. The experiments were performed in three phases [11].

(1) In the preliminary experiments, the round trip propagation time between the ground and the GEO satellite was measured. Then, it was compared with the estimated propagation time that was calculated beforehand with the simulators. The estimation error was confirmed to be from $4,700 \mathrm{~ns}$ to $4,800 \mathrm{~ns}$ in all experiments and was due mostly to instrumental constants.

(2) In the uplink experiments, OCXO 8607 was controlled only with the feed-forward loop of the ground site. We confirmed that the use of the crystal oscillator controller resulted in successful convergence of the OCXO 8607 phase to the offset configured beforehand. However, since OCXO 8607 was steered

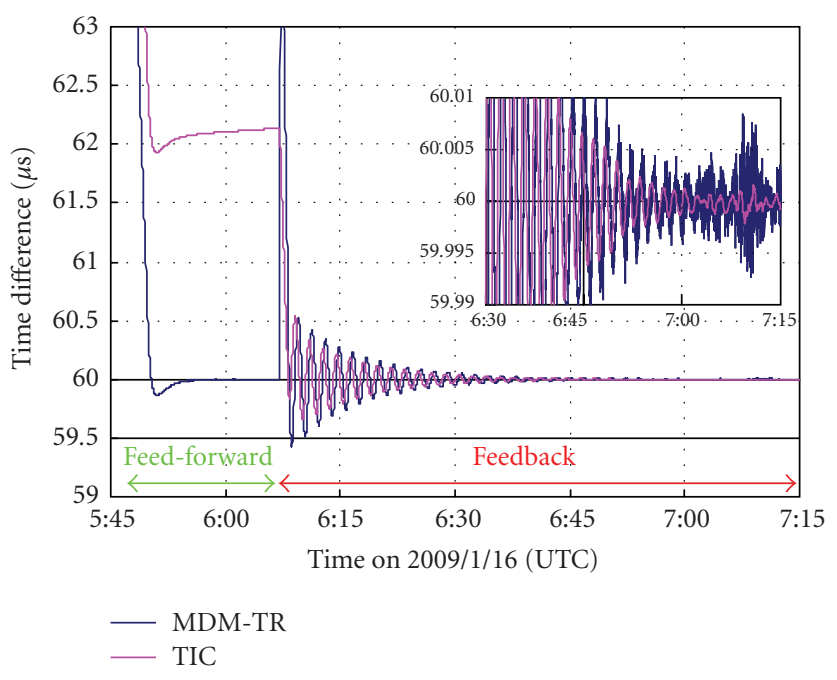

FIgURE 14: Results of final feedback experiment.

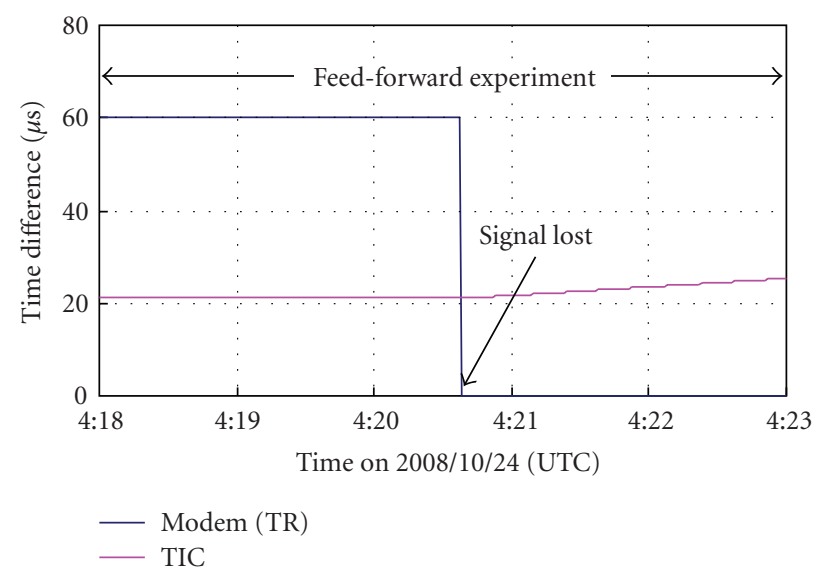

Figure 15: Experimental data showing signal loss due to precipitation.

to eliminate clock error as well as the estimation error observed in the previous experiment, the time difference between OCXO 8607 and the $\mathrm{H}$-maser observed in TIC was confirmed to be close to the estimation error calculated afterwards.

(3) In the end-to-end experiments of RESSOX, OCXO 8607 was controlled by a combination of feedforward and feedback loops. The estimation error that remained in the clock error in the previous experiments was confirmed to be reduced by feedback control. However, since the delays caused by the passage of the uplink and downlink signals through different media were not perfectly estimated in the experiment, they remained as the offset of $32 \mathrm{~ns}$ in the clock error. Therefore, numerical simulation was conducted with consideration of the error sources, and it was confirmed that OCXO 8607 was synchronized to the $\mathrm{H}$-maser within an error of $10 \mathrm{~ns}$. 


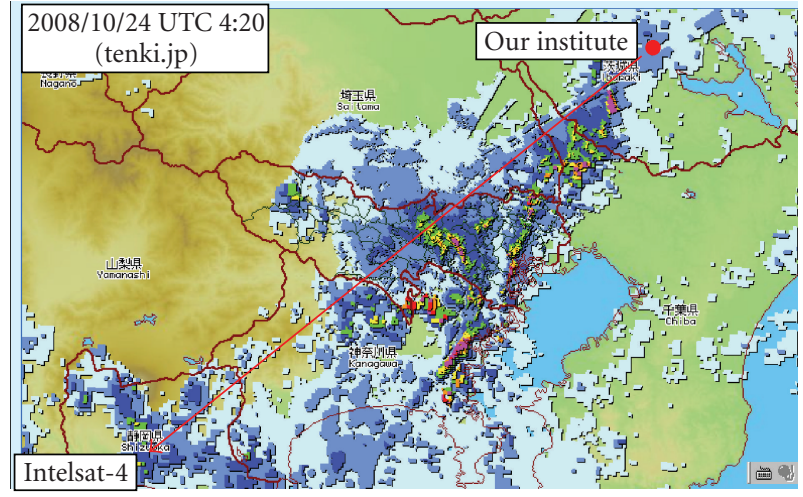

FIgURE 16: Precipitation data from a website.

3.4. Preliminary Ground Experiments for Intelsat-4. Before the experiments using Intelsat-4, preliminary ground experiments were conducted. The experimental setup is presented in Figure 5. A delay simulator that causes $0.01 \mathrm{~s}$ delay is used for downlink simulation, because modem (R) does not work when the time difference is smaller than $0.006 \mathrm{~s}$.

3.4.1. Feed-Forward Control Experiments. To simulate feedforward control, TTA was set to zero-second advance and OCXO 8607 was controlled to make the time difference between OCXO 8607 and uplink signals $60 \mu$ s (since TIC displays only positive values, a synchronization offset was given). The result is shown in Figure 6. The time difference measured with modem (TR) was controlled to exactly $60.0 \mu \mathrm{s}$; however, the time difference measured with TIC was $56.9760 \mu \mathrm{s}$. This means that TTA has an internal delay of $3.0240 \mu \mathrm{s}$.

To compensate the internal delay of TTA, TTA advanced the $\mathrm{H}$-maser time by $3.0240 \mu \mathrm{s}$. The result is shown in Figure 7. The time difference measured with TIC coincides with that measured with modem (TR). This means that the feed-forward control in which TTA advances H-maser time by $3.0240 \mu$ s is successful.

3.4.2. Feedback Control Experiments. Next, experiments of feedback control, which compensates the feed-forward control error, were executed on the ground. As shown previously, there is no error in the feed-forward control in which $\mathrm{H}$ maser time is advanced by $3.0240 \mu$ s, and this calibration was conducted throughout the experiments. The result is shown in Figure 8. First, as the feed-forward command, 30 ns error was given ((a) it means that the distance between the QZS and the ground station was overestimated by $9 \mathrm{~m}(=30 \mathrm{~ns}))$. Therefore, in the feed-forward phase, the time difference was $30 \mathrm{~ns}$. Then, feedback control was conducted. As the feedback adjustment, the command of 30 ns delay was given. As a result, the feed-forward control error was cancelled. Second, the feed-forward control error of uplink delay, zero at $0 \mathrm{~s}$, and the rate of change of $1 \mathrm{~ns} / \mathrm{s}$ were used (light blue line in Figure 8(b)). When only feed-forward control was executed, the time difference followed the feed-forward command, which means that the pink line corresponds to the light blue line. Then, when feedback control was executed, the pink line converged to $60 \mu \mathrm{s}$.

3.5. Experiments Using Intelsat-4. Intelsat-4 was launched on August 3, 1995, from Guiana on the Ariane 4 rocket, and is located at 72 degrees east longitude. The elevation angle from our institute is 7.9 degrees, so that large tropospheric delay is expected.

3.5.1. Feed-Forward Control Experiments. To conduct the feed-forward experiments, transmitting delay was estimated to be approximately $0.1357 \mathrm{~s}$ (one way) on the basis of the orbit information of Intelsat-4. The following are the parameters of the orbit for delay estimation: semimajor axis $=42166.28 \mathrm{~km}$, eccentricity $=0.0003377$, inclination $=$ 0.01514 degrees, RAAN $=209.0799$ degrees, argument of perigee $=350.9648$ degrees, and mean anomaly $=258.1149$ degrees True of Date at 2008/10/20 23:45:12 in UTC. Figure 9 shows the results of feed-forward experiments. For the delay estimation, transmitting delay (approximately $0.2714 \mathrm{~s}$ round trip) and TTA internal delay $(3.0240 \mu \mathrm{s})$ were considered. Our target was a $60 \mu$ s advancement of OCXO 8607 with the H-maser, and the total delay was advanced at TTA because TIC measures only positive delay. However, the obtained time difference measured by TIC was approximately $21.3 \mu$ s (approximately $38.7 \mu$ s difference). It is surmised that the origin of the difference comprises the internal delays of components such as the up-converter, the down-converter, cables, and antennas, and the error of satellite orbit estimation.

3.5.2. Feedback Control Experiments. Next, feedback control experiments were conducted. Feedback command is generated on the basis of the difference between measured time delay and estimated time delay. The difference is named the time to be adjusted and includes range error, clock error and tropospheric delay. Feedback command was extrapolated with the first-order least-mean-squares filter using one hundred data values (from six seconds ago to 105 seconds ago for every second) of time to be adjusted, as shown in Figure 10. Figure 11 shows the results of feedback experiments. The parameters of the orbit used for delay estimation are semimajor axis $=42165.11 \mathrm{~km}$, eccentricity $=0.00029187$, inclination $=0.02181$ degrees, RAAN $=278.9775$ degrees, argument of perigee $=16.0096$ degrees, and mean anomaly $=290.6198$ degrees True of Date at 2009/1/15 2:35:27 in UTC. The TTA uplink signal advanced the standard time by the sum of transmitting delay (approximately $0.2714 \mathrm{~s})$, TTA internal delay $(3.0240 \mu \mathrm{s})$, and other estimated delays $(38.7 \mu \mathrm{s})$. In the case of the feed-forward phase, an approximately $1.5 \mu$ s delay was still observed (left side of Figure 11) as a result of orbit estimation error. In the feedback phase, oscillation continued for more than 30 minutes, and this is considered as ringing in feedback control. We confirmed that the number of time values to be adjusted for feedback control (in our case, the number is 100) affects the ringing time; the more 
the number of time values to be adjusted, the longer the ringing time. In the feedback phase, most of the feedforward error was cancelled; however, a 2 ns offset of the time difference remained (right side of Figure 11; Figure 12 shows a magnified figure). Although 2 ns offset is acceptable for our target, the origin of this offset was investigated.

Figure 13 shows the block diagram of loop delay measurements between the uplink part (A) and the downlink part (B). A time difference of approximately $4 \mathrm{~ns}$ between A and $\mathrm{B}$ was observed.

Finally, the feedback experiment in which the difference between loop delays (approximately $4 \mathrm{~ns}$ ) was considered was conducted. The result is shown in Figure 14. The offset disappeared and the total error was reduced to almost zero. This means that even under a low-elevation-angle condition that includes considerable tropospheric delay, it was confirmed that the feedback control of RESSOX was effective.

3.6. Precipitation Attenuation Effects. Since precipitation attenuates the $\mathrm{Ku}$ band $(12-15 \mathrm{GHz})$, particularly at a low elevation angle, our experiment was unintentionally interrupted. Figure 15 shows experimental data that indicate signal loss due to precipitation, and Figure 16 shows the precipitation data at that time, obtained from a meteorological website. Figure 16 indicates that there was much precipitation between Intelsat-4 and the ground site during the experiment.

This means that precipitation requires special attention at low elevation angles when the QZSS is controlled by RESSOX.

\section{Conclusions}

This study is summarized as follows.

(1) The apparatuses used in this research were introduced.

(2) Feed-forward and feedback control results of preliminary ground experiments were presented. Feedforward control worked well when TTA delay $(3.0240 \mu \mathrm{s})$ was compensated. Feedback control worked well and compensated the time offset and the delay change rate.

(3) The results of feed-forward and feedback control experiments using Intelsat- 4 were described. In the feed-forward experiment, estimation delay error that includes the error of cables was $38.7 \mu \mathrm{s}$. When the time difference due to loop delay between uplink and downlink (4 ns) was considered, the feedback error was almost zero nanosecond.

(4) Signal loss due to precipitation was observed. Special attention should be paid to precipitation at low elevation angles when the QZSS is controlled by RESSOX.

\section{Acknowledgments}

This study was carried out as part of the "Basic Technology Development of Next-Generation Satellites" project promoted by the Ministry of Economics, Trade and Industry (METI) through the Institute for Unmanned Space Experiment Free Flyer (USEF).

\section{References}

[1] M. Kishimoto, H. Hase, A. Matsumoto, T. Tsuruta, S. Kogure, N. Inaba, M. Sawabe, T. Kawanishi, S. Yoshitomi, and K. Terada, "QZSS system design and its performance," in Proceedings of the Institute of Navigation National Technical Meeting, pp. 405-410, 2007.

[2] J. J. Suter, L. J. Crawford, B. G. Montgomery, and W. E. Swann, "Syntonics LLC: APL-developed technology makes its commercial debut," Johns Hopkins APL Technical Digest, vol. 22, no. 2, pp. 168-175, 2001.

[3] P. A. Koppang, D. Matsakis, and M. Miranian, "Alternate algorithms for steering to make GPS time," in Proceedings of 13th International Technical Meeting of the Satellite Division of the Institute of Navigation (ION GPS '00), pp. 933-936, 2000.

[4] D. W. Allan, N. Ashby, and C. C. Hodge, The Science of Timekeeping, Application Note 1289, Hewlett Packard, 1997.

[5] D. Giunta, G. Busca, A. Della Torre, M. Vitta, V. De Perini, R. Grimoldi, X. Stehlin, and P. Rochat, "Recent developments in time \& frequency dissemination systems," in Proceedings of the 18th European Frequency and Time Forum (EFTF' 04), pp. 530-536, 2004.

[6] F. Tappero, A. Dempster, T. Iwata, et al., "Proposal for a novel remote synchronization system for the on-board crystal oscillator of the quasi-zenith satellite system," Navigation, vol. 53, no. 4, pp. 219-229, 2006.

[7] T. Iwata, M. Imae, T. Suzuyama, et al., "Simulation and ground experiments of remote synchronization system for onboard crystal oscillator of quasi-zenith satellite," Navigation, vol. 53, no. 4, pp. 231-235, 2006.

[8] T. Iwata, Y. Kawasaki, M. Imae, T. Suzuyama, T. Matsuzawa, S. Fukushima, Y. Hashibe, N. Takasaki, K. Kokubu, A. Iwasaki, F. Tappero, A. Dempster, and Y. Takahashi, "Remote synchronization system of quasi-zenith satellites using multiple positioning signals for feedback control," Navigation, vol. 54, no. 2, pp. 99-108, 2007.

[9] T. Iwata, M. Imae, T. Suzuyama, et al., "Remote synchronization simulation of onboard crystal oscillator for QZSS using L1/L2/L5 signals for error adjustment," International Journal of Navigation and Observation, vol. 2008, Article ID 462062, 7 pages, 2008.

[10] H. Kiuchi and T. Kondo, "The wavefront clock technique applied to current VLBI systems," Publications of the Astronomical Society of Japan, vol. 48, no. 1, pp. 137-146, 1996.

[11] N. Takasaki, A. Iwasaki, T. Iwata, M. Imae, and T. Suzuyama, "Remote clock synchronization using geostationary satellite for Japanese quasi-zenith satellite system," in Proceedings of the 19th International Technical Meeting of the Satellite Division of the Institute of Navigation (ION GNSS '06), pp. 2120-2130, 2006. 

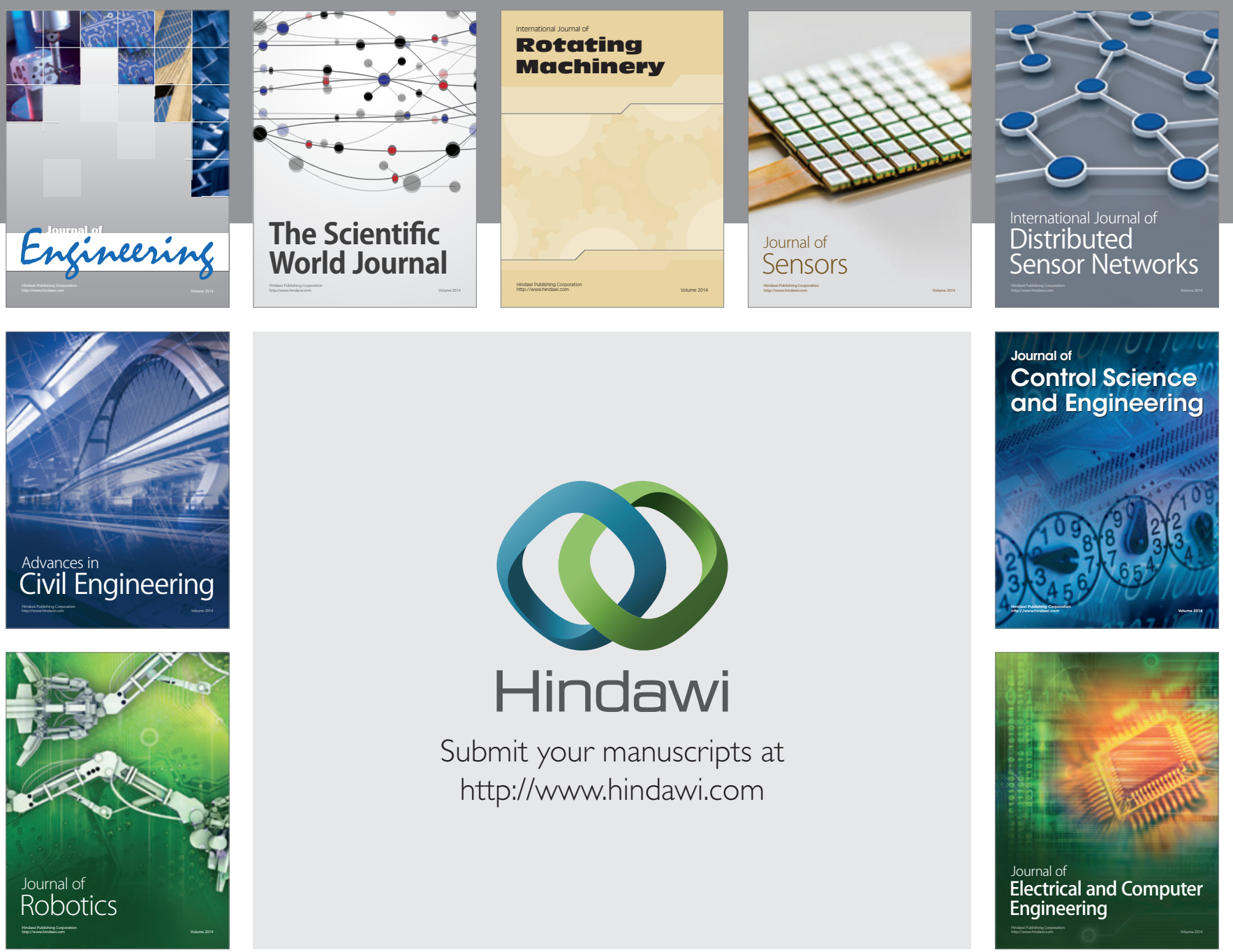

Submit your manuscripts at

http://www.hindawi.com
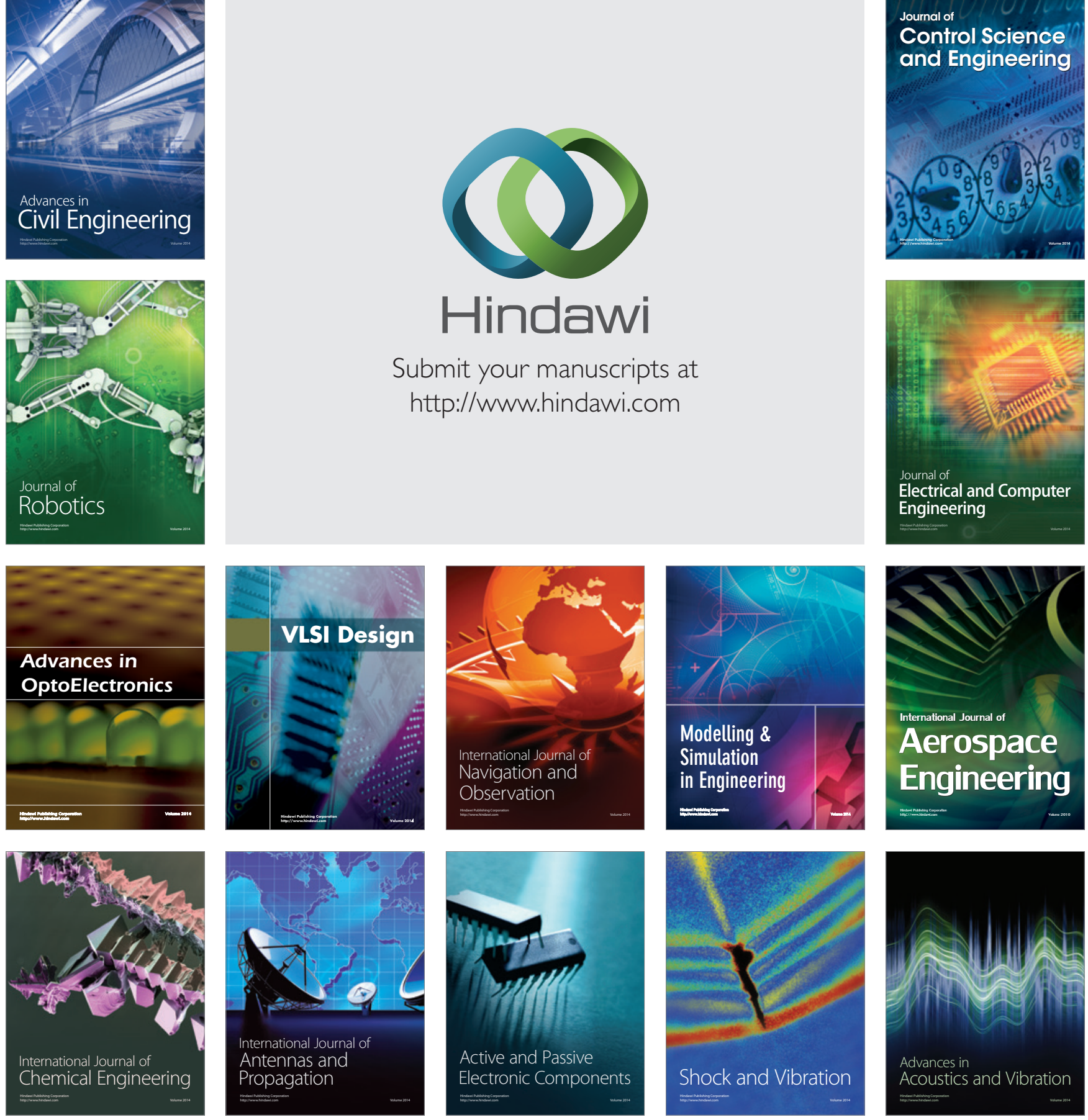